\title{
Stochastic reconstruction of filament paths in fibre bundles based on two- dimensional input data
}

F. Gommer $^{* 1}$, K. Wedgwood ${ }^{2}$, L.P. Brown ${ }^{1}$

1) Polymer Composites Group - Division of Materials, Mechanics \& Structures,

2) School of Mathematical Sciences

University of Nottingham, University Park, Nottingham NG7 2RD, UK

* Corresponding author (F.Gommer@nottingham.ac.uk)

Abstract: Conventional optical microscopy is an inexpensive technique to analyse fibre bundle micro-structures. Compared to micro-computed tomography, the resolution is higher and larger samples sizes can be analysed. This provides, for example, detailed information of filament spacing which will affect subsequent properties. This paper proposes a probabilistic method to reconstruct paths of individual filaments from a series of two-dimensional micrographs. This allows three-dimensional filament arrangements to be reconstructed and the effects of local arrangements on properties to be quantified. For example, for the small sample analysed in this work, a $15 \%$ difference in strength values was found.

Keywords: A. Carbon fibre, B. Microstructures, C. Analytical modelling, D. Optical microscopy

\section{Introduction}

Textile fibre reinforcements used in composites are usually made from filaments bundled into tows. The fibre bundles within fabrics are not straight but exhibit in- [1] and out-of-plane waviness [2]. It was shown that such waviness can significantly affect the resulting properties of a composite $[3,4]$. In addition to the waviness of an entire fibre bundle, the filaments within bundles also exhibit undulation and twist [5]. These 
filaments do not necessarily follow the path of the bundle exactly and adjacent filaments are not necessarily parallel to each other. These additional misalignments on the microscale will affect the composite properties locally, for example promoting void formation during impregnating flow [6], which will have an adverse effect on the mechanical properties [7]. Local effects can lead to filament fracture which may cause crack growth and failure [8].

For numerical predictions of composite properties, the filaments in fibre bundles are usually assumed to be straight parallel rods, arranged in a periodic [9] or random [10] pattern. This allows simplification of model domains to two-dimensions. The assumption of parallel alignment of filaments may be applicable to small sections of a fibre bundle. In reality, the filaments exhibit random waviness which will gradually change the filament arrangement along the path of a fibre bundle [5]. This causes distances between neighbouring filaments to vary, which in turn affects local flow [11] or mechanical [12] properties.

It is possible to use micro-computed-tomography (micro-CT) to visualise the random three-dimensional filament arrangement (Figure 1); however, these studies are limited to small areas only $[5,13]$. As a rule of thumb for current 'off-the-shelf' systems, the maximum sample size to be scanned in $\mathrm{mm}$ is equivalent to the achievable scan resolution in $\mu \mathrm{m}$. Tiling of volume images and movable detectors allow larger samples to be analysed at high resolution. The scan times do, however, increase significantly (up to days). In addition, thicker samples will also increase the problem of artefacts occurring during the scan [14]. The current achievable resolution of commercially available micro-CT systems is limited to a maximum of about $0.5 \mu \mathrm{m}$ and can be improved to an order of about $0.1 \mu \mathrm{m}$ for laboratory systems [15]. This makes 
determination of exact positions and shapes of individual filaments difficult. Hence, it is not possible to measure small gaps between closest filaments, which are often below this resolution limit [16]. In the case of carbon fibre reinforced composites, the resulting volume images do not exhibit good contrast due to the low x-ray absorption of the fibres [17] and image analysis is therefore often limited to manual identification of features [2, 18]. Automated analysis methods using anisotropy filters can overcome this problem $[19,20]$.

Traditional techniques such as optical microscopy [21] or scanning electron microscopy (SEM) [22] can be used to achieve images of cross-sections at a higher resolution. Image tiling and automated image analysis also make it feasible to study larger areas of a material [23]. For example, to scan an entire fibre bundle cross-section, approximately 1 to 45 minutes are needed depending on the resolution and sample quality. However, as these techniques analyse surfaces only, a larger amount of sample preparation is required which increases the risk of damage compared to micro-CT. In addition artefacts due to, for example, lens distortions need be considered and, if necessary, be corrected.

This paper proposes a probabilistic methodology based on the Hungarian (or Munkres) algorithm [24], which allows reconstruction of filament paths from two-dimensional micrographs of slices of a material. A more simplistic approach using very small distances between individual slices and direct mapping of points has, for example, been used to reconstruct the shape of a fibre bundle [25] or the filament arrangement in a small sample [26]. In this work, a series of micrographs of a carbon fibre bundle crosssection presented by Mansfield [27], cut perpendicular to the nominal filament direction and taken after repeatedly removing small amounts of material at the surface, are 
analysed. Determined cross-sectional centre points of the filaments in different micrographs allow prediction of which points are connected in different slices. The open source software TexGen [28] is then used to fit a spline through the different measurement points which enables reconstruction of three-dimensional filament paths. The stochastic approach used in this work is expected be more robust than simple assignment of closest points in micrographs which requires very small distances, e.g. 20 $\mu \mathrm{m}$, between slices [26]. The disadvantage of this reconstruction method being destructive is compensated by the higher image resolution and the larger sample surface which can be studied at a significantly lower cost. This measurement technique will allow larger amounts of material to be studied which in turn will help to better understand the mechanics within fibre bundles using standard inexpensive analysis techniques. Using higher magnification images in the future will also allow interpolation of exact filament shapes in, for example, a 12k fibre bundle (Figure 2).

\section{Materials and data acquisition}

The data used in this work were acquired from Mansfield's publication [27]. A small section of carbon fibre reinforced composite of approximately $100 \mu \mathrm{m} \times 140 \mu \mathrm{m}$, cut perpendicular to the nominal fibre path (Figure 3), was analysed by means of Scanning Electron Microscopy (SEM). The average filament diameter, $d$, in this material was estimated to be about $8 \mu \mathrm{m}$. Subsequently, small amounts of material $(25,60,100,150$, $180 \mu \mathrm{m})$ were removed by abrasion and the sample surfaces were repeatedly examined. This made it feasible to analyse the cross-sectional positions of fibres throughout the material. Based on a fixed reference point inside the sample and the shape of selected single fibre cross-sections, Mansfield was able to evaluate changes in position of selected filament cross-sections manually. However, due to the amount of work, he only 
analysed a small number of filaments which could be unequivocally identified. These filament cross-sections in different slices were used to validate the probabilistic point matching approach in this work.

As the images used are reproduced from printed literature [27], their quality is poor. Hence, it is not possible to accurately determine filament edges as it would be possible from the original images [16]. To demonstrate the feasibility of the matching algorithm in this work it is, however, sufficient to use the centre points of the filament crosssections only (Figure 3). The available image quality still allows clear differentiation between densely packed filaments which may not be possible when using micro-CT (Figure 1C). The centre positions for 128 filaments are determined from fitting ellipses through a number of manually selected points at their edges.

\section{Point matching algorithm}

In order to construct the $3 \mathrm{D}$ approximations to the filaments, points in the cross sections need to be matched to points in each of the other sections. This falls into the class of assignment problems, commonly seen for example in job allocation [29-31] and target tracking. In the computer science literature, this is often referred to as the "knapsack problem" in which $n$ balls have to be placed into $m$ knapsacks. A review of the problem, together with common algorithms to solve it is given by Poore and Gadaleta [32]. Placing a ball in a knapsack incurs some cost or penalty and each knapsack has a maximum weight of balls that it can hold. The aim is then to place the balls in the knapsacks so as to minimise the total cost accrued. In general, since the aim is to reconstruct the full fibres from the cross sections, all possible paths that pass through exactly one point in each of the cross sections should be considered. The computational cost associated with performing this task is great. In this initial study, the approach is 
instead to match points between adjacent cross sections under the assumption that filaments are 'well-behaved'. This presumes that filament paths do not change abruptly and are continuous. This greatly reduces the number of paths to consider, and the problem boils down to exactly that of the knapsack problem, which is an example of a generalised assignment problem. In terms of complexity, this problem is $N P$-hard [33] and thus solving it is still computationally demanding.

Approaches have involved formalising the problem as a bipartite graph between the balls and the knapsacks and searching for minimal augmenting paths [34] between the two or random search algorithms in the form of particle swarm optimisation [35]. Linear assignment problems, in which the number of balls and the number of knapsacks are equal, can be solved exactly, incurring a reasonable computational cost.

Given two adjacent cross-sections, $A$ and $B$, the centre points in section $A$ correspond to the balls in the knapsack problem and their equivalents in section $B$ are the knapsacks. In the analysis, one point per cross section is allocated per filament. To convert the filament reconstruction problem to a linear assignment problem, only the filaments that could be fully resolved in each of the sections were used to approximate the fibres. The problem is now to assign each point in section $A$ to one and only one point in section $B$, so as to minimise some yet to be defined cost, and then repeat this for sections $B$ and $C$, and so on. The maximum distance between adjacent cross-sections to accurately reconstruct filament paths in practise does, of course, depend on the expected maximum amount of variability in filament paths.

Following this, the $n$ constructed filaments will then pass through each section transversally at exactly one point. The hope is then that the paths constructed in this recursive manner also minimise the equivalent cost when considering all sections 
simultaneously. This problem can be solved via the Hungarian algorithm [24, 36, 37], which has been proven to find the exact solution in order $\mathrm{O}\left(n^{3}\right)$ time. Extensions to the Hungarian algorithm can deal with so-called rectangular problems, which do not require the same number of points in each section.

\subsection{Cost function}

Assigning costs to each of the possible assignments between two slices is done through a cost function. The simplest possible way to do this is to use the Euclidean distance between points. This requires aligning the two slices under consideration so that they share a common origin. To simplify matters further, the $z$ component of the distance can be excluded, so that the distance under consideration assumes that points in one slice are projected onto the next. Were filaments truly arranged in rod-like fashion, perpendicular to the cross sections, the distance between correctly assigned points would be zero. When only the filament centres are available, Euclidean distance is the most appropriate cost function to choose. Since Mansfield's analysis of the filaments [27], better imaging methods have allowed for the better resolution of the ellipsoid that are used to identify the filaments in the cross sections [16]. The better approximations provide more information to characterise the filament paths. In particular, the eccentricity of the ellipse may be used to find the angle that the filament makes with the cross section. This knowledge can be used to better approximate the filament's path to the next cross section.

Under the assumption that the path of a filament between cross sections is simply a straight line, its most likely intersection with the next cross section can be computed, given the location and eccentricity of the previous intersection. Note that this gives two possible intersection points in the next slice since the eccentricity of the filament 
through the cross section gives two possible angles (Figure 4), however, we can use knowledge of previous assignments to restrict this to just one possibility. Of course, these paths are not true straight lines, and so these points are then used as a mean for a probability distribution aimed at capturing the deviation of the path from a straight line. This uncertainty is captured by a bivariate Gaussian distribution whose covariance is chosen to align with the angle as given by the eccentricity, and scaled to reflect both some intrinsic degree of uncertainty as well as the distance between cross sections (Figure 5). The scale of the variance of the Gaussian distributions is a parameter that may be altered when performing the assignment. More sophisticated methods can use the Kalman filter [38-40] to modify the covariance of these distributions as assignments are recursively made between cross sections.

For the case in which ellipses cannot be resolved accurately, and only the centre points are available, these Gaussian distributions are chosen to have a covariance given by a scaled version of the identity matrix. For each point in a given slice, the points on the next slice then have a probability of being matched to the initial point based on these distributions. The cost function is then given by the reciprocal of the sum of these probabilities, so that minimising the cost corresponds to maximising the probability that two points on adjacent cross sections share a filament (Figure 6). If $P_{i j}$ is the probability that the centre point $i$ in cross section $A$ is part of the same filament as centre point $j$ in cross section $B$, then the cost function between the two cross sections is

$$
\operatorname{Cost}(A, B)=\frac{1}{\sum_{i j} P_{i j}^{A B}}, \quad P_{i j}^{A B}=\frac{1}{2 \pi \sigma_{A B}^{2}} \exp \left\{-\frac{\left(x_{i}^{A}-x_{j}^{B}\right)^{2}}{2 \sigma_{A B}^{2}}-\frac{\left(y_{i}^{A}-y_{j}^{B}\right)^{2}}{2 \sigma_{A B}^{2}}\right\}
$$

where $\left(x_{i}^{A}, y_{i}^{A}\right)$ are the coordinates of centre point $i$ in cross section $A$ and $\sigma_{A B}$ is the standard deviation of the bivariate Gaussian distribution, which is directly proportional 
to the distance between the two cross sections. In future work, cost functions will be given using the full method described above, in which $P_{i j}$ are replaced with $P_{i j}=\frac{1}{2 \pi \sigma_{i}^{x} \sigma_{i}^{y} \sqrt{1-\rho_{i}^{2}}} \exp \left\{-\frac{z_{i j}}{2\left(1-\rho_{i}^{2}\right)}\right\}, \quad z=\frac{\left(\hat{x}_{i}-x_{j}\right)^{2}}{\left(\sigma_{i}^{x}\right)^{2}}+\frac{\left(\hat{y}_{i}-y_{j}\right)^{2}}{\left(\sigma_{i}^{y}\right)^{2}}+\frac{2 \rho_{i}\left(\hat{x}_{i}-x_{j}\right)\left(\hat{y}_{i}-y_{j}\right)}{\sigma_{i}^{x} \sigma_{i}^{y}}$ In the above, in which the $A B$ dependence is suppressed, the contribution of the variance is now split into its $x$ and $y$ components, whilst $\rho$ is the correlation between these two. The variables $\left(\hat{x}_{i}, \hat{y}_{i}\right)$ correspond to the projection of a supposed path through $\left(x_{i}^{A}, y_{i}{ }^{\mathrm{A}}\right)$ onto cross section $B$. This projection is done by first fitting a straight line through $\left(x_{i}^{A}, y_{i}\right.$ A) in cross section $A$ whose slope is determined via the eccentricity of the ellipse representing the filament section and then by examining this line's intersection with cross section $B$.

\subsection{The algorithm}

The first step in the Hungarian algorithm is forming a $n \times n$ matrix whose entries are given between the cost of assigning points in section $A$ to those in $B$. In this matrix, the rows correspond to points in section $A$, whilst the columns correspond to points in section $B$. For computational efficiency, certain assignments can be excluded from consideration. One way of doing this is to define a search radius around the point in section $A$ (Figure 6). This can either be treated as a free parameter, or a threshold condition can be applied so that the radius is defined to be the point at which the probability of connection between points in adjacent cross sections falls below some set value. Assignments that would connect this point in section $A$ to point in section $B$ outside this search radius are then prohibited. In terms of the cost matrix, this can be done by setting the cost outside of the search radius to have infinite value.

Once the cost matrix has been defined, an assignment consists of picking, for each row 
$i$, a column $j$. The cost for that allocation is simply given by the value of the matrix element $\mathrm{M}_{i j}$, and thus the total cost for the assignment is the summation of all of the individual costs. Finding optimal assignments for a given cost matrix by inspection is difficult. Fortunately, there is a useful theorem that can be utilised to reduce the problem. If a number is added to or subtracted from all of the entries of a row or column of a cost matrix, then an optimal assignment for the resulting cost matrix is an optimal assignment for the original matrix. The Hungarian algorithm uses these observations to recursively reduce the original cost matrix until an assignment can be made all of whose individual assignments have zero cost. Since all costs are positive, this represents an optimal assignment. The algorithm thus follows these steps:

Step 1: Subtract the smallest entry from each row from all of the entries of its row.

Step 2: Subtract the smallest entry from each column for all of the entries of its column.

Step 3: Draw lines through the rows and columns of the matrix so that all of the zeros are covered by the lines. This should be done only by drawing lines either through the entirety of one row or the entirety of one column and by using the fewest possible lines.

Step 4: If the number of lines drawn is equal to the number of rows in the matrix, then the optimal assignment has been found. If the number of lines is not equal to the number of rows, then proceed to step 5.

Step 5: Subtract the smallest entry not covered by any of the lines from each uncovered row, then add it to each covered column, then, return to step 3.

A Matlab implementation of the Hungarian algorithm can be found in [41], and this is the one used in the present study. The original algorithm works in $\mathrm{O}\left(n^{4}\right)$ time, but 
improvements can be made so that it can be run in $\mathrm{O}\left(n^{3}\right)$ time. As the number of elements scales, the computational cost thus increases cubically. In addition, the memory required to store the cost matrix increases quadratically. Using a standard desktop computer with 8GB memory, it was found to be feasible to match 12000 points and therefore feasible to reconstruct a 12k fibre bundle such as shown in Figure 1 from individual micrographs.

Since many of the entries of the cost matrix represent allocations that are not allowed, the assignment problem is sparse and so other approaches can be used. One such example, due to Jonker and Volgenant [34] constructs minimally augmenting paths to provide the optimal assignment. One property of such methods is that the construction of the full cost matrix can avoided. In addition, these methods can be extended to deal with the generalised assignment problem [42], where the number of filaments through each cross section is not the same.

\subsection{Hungarian example}

To illustrate how the Hungarian algorithm works in practice, an example of a point matching between two sections, each containing five filaments is shown below, as depicted in Figure 7. The row entries of the cost matrix are generated by first centring a bivariate Gaussian with an identity covariance matrix on a point in section $A$, and then by taking the reciprocal of the probabilities given by these distributions evaluated at points in $B$. The cost matrix for this problem is then

$M=\left[\begin{array}{rrrrr}12.6 & 20.9 & 629.2 & 82.7 & 30.7 \\ 21.8 & 12.6 & 121.6 & 59.2 & 69.1 \\ 690.6 & 149.6 & 13.1 & 22.9 & 163.6 \\ 86.0 & 24.6 & 20.2 & 24.2 & 86.3 \\ 31.8 & 36.9 & 77.4 & 16.4 & 14.3\end{array}\right]$

The result of steps 1 and 2 of the algorithm lead to 
Step 1: $\left[\begin{array}{ccccc}12.6 & 20.9 & 629.2 & 82.7 & 30.7 \\ 21.8 & 12.6 & 121.6 & 59.2 & 69.1 \\ 690.6 & 149.6 & 13.1 & 22.9 & 163.6 \\ 86.0 & 24.6 & 20.2 & 24.2 & 86.3 \\ 31.8 & 36.9 & 77.4 & 16.4 & 14.3\end{array}\right] \rightarrow\left[\begin{array}{ccccc}0 & 8.3 & 616.6 & 70.1 & 18.1 \\ 9.2 & 0 & 109.0 & 46.6 & 56.5 \\ 677.4 & 136.5 & 0 & 9.7 & 150.5 \\ 65.7 & 4.3 & 0 & 4.0 & 66.1 \\ 17.5 & 22.5 & 63.1 & 2.1 & 0\end{array}\right]$

Step 2: $\left[\begin{array}{ccccc}0 & 8.3 & 616.6 & 70.1 & 18.1 \\ 9.2 & 0 & 109.0 & 46.6 & 56.5 \\ 677.4 & 136.5 & 0 & 9.7 & 150.5 \\ 65.7 & 4.3 & 0 & 4.0 & 66.1 \\ 17.5 & 22.5 & 63.0 & 2.0 & 0\end{array}\right] \rightarrow\left[\begin{array}{ccccc}0 & 8.3 & 616.6 & 68.0 & 18.1 \\ 9.2 & 0 & 109.0 & 44.5 & 56.5 \\ 677.4 & 136.5 & 0 & 7.6 & 150.5 \\ 65.7 & 4.3 & 0 & 1.9 & 66.1 \\ 17.5 & 22.5 & 63.1 & 0 & 0\end{array}\right]$

One example of the covering produced by step 3 is

Step 3: $\left[\begin{array}{ccccc}0 & 8.3 & 616.6 & 68.0 & 18.1 \\ -9.2 & 0 & 109.0 & 44.5 & 56.5 \\ 677.4 & 136.5 & 0 & 7.6 & 150.5 \\ 65.7 & 4.3 & 0 & 1.9 & 66.1 \\ 17.4 & 22.5 & 63.1 & 0 & 0\end{array}\right]$

All of the zeros can be covered with four lines, which is fewer than the number of

filaments so, according to step 4, step 5 must be followed.

Step 5: $\left[\begin{array}{ccccc}0 & 8.3 & 618.6 & 68.0 & 18.1 \\ 9.2 & 0 & 109.0 & 44.5 & 56.5 \\ 675.5 & 134.5 & -1.9 & 5.7 & 148.6 \\ 63.8 & 2.4 & -1.9 & 0 & 64.2 \\ 17.5 & 22.5 & 63.0 & 0 & 0\end{array}\right] \rightarrow\left[\begin{array}{ccccc}0 & 8.3 & 618.5 & 68.0 & 18.1 \\ 9.2 & 0 & 110.9 & 44.5 & 56.5 \\ 675.5 & 134.5 & 0 & 5.7 & 148.6 \\ 63.8 & 2.4 & 0 & 0 & 64.2 \\ 17.5 & 22.5 & 65.0 & 0 & 0\end{array}\right]$

The amended cost matrix becomes

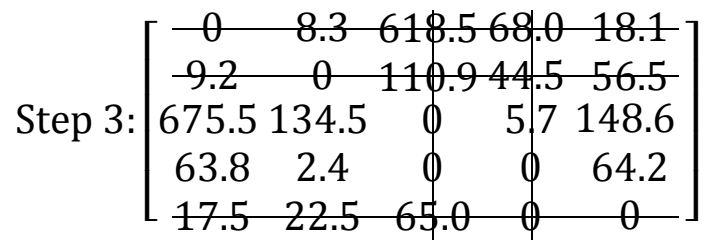

The zeros are then covered by a minimum of five lines and thus the optimal assignment has been found (Figure 7B), as shown by the highlighted entries.

$$
\left[\begin{array}{ccccc}
0 & 8.3 & 618.5 & 68.0 & 18.1 \\
9.2 & 0 & 110.9 & 44.5 & 56.5 \\
675.5 & 134.5 & 0 & 5.7 & 148.6 \\
63.8 & 2.4 & 0 & 0 & 64.2 \\
17.5 & 22.5 & 65.0 & 0 & 0
\end{array}\right]
$$




\section{Reconstructed micro-structure}

Determining the probability of filament cross-sections to be connected in micrographs taken at subsequent positions along the path of a fibre bundle makes it feasible to reconstruct paths of individual filaments. Using the Hungarian algorithm (Section 3), it was possible to determine the connectivity between filaments in the six micrographs presented by Mansfield [27]. This was achieved by subsequently matching the filament centre points in adjacent slices. The accuracy of the obtained results was validated with the set of marked filaments manually matched by Mansfield.

In order to analyse the influence of distance between micrograph sections on the matching algorithm, slices were matched by leaving out intermediate sets of data. This made it feasible to determine a distance limit, $l$, of about $0.3 \mathrm{~mm}(l / d \approx 22)$ between micrographs for accurate matching of points. Larger distances were found to result in incorrect filament paths. This is significantly larger than the previously used $0.02 \mathrm{~mm}$ [26]. It may even be possible to increase it further when using more information such as filament cross-sectional shapes during the matching process which may allow paths of strongly curved filaments (Figure 1) to be accurately determined as well. The maximum distance between micrographs in practice will depend on the expected waviness and the local density of filaments, which makes it challenging to estimate.

The filament centre points in different slices were assigned constant circular crosssections which were chosen to ensure a sufficiently large gap between individual filaments for discretisation of the model domain in to finite elements. The open source software TexGen [43] was used to extrude the fitted cross-sectional shapes along a best fit spline connecting specified centre points. This results in a three-dimensional geometric model of the filament arrangement for the analysed fibre bundle section 
(Figure 8). These models can, for example, be used for numerical modelling of the properties of the filament arrangement.

\section{$5 \quad$ Influence of fibre misalignment}

It is currently not feasible to discretise the geometry shown in Figure 8 into a form suitable for use in a numerical solver. To gain an estimate of the influence of filament misalignment on the mechanical properties, an analytical solution is used in this work. Using the Python scripting interface of TexGen [28], it is possible to extract information of individual filament orientations at any position along their path. These local orientation vectors determine the alignments, $\theta$, of the filaments in respect to the nominal coordinate system of the model (Figure 9). These angles can be used to analytically determine the influence of misalignment on the properties of individual filaments. This is demonstrated on stiffness and strength distributions of the reconstructed bundle section. The angles as a function of position along the filament paths show a large negative peak at $-25 \mu \mathrm{m}$ (for the second image). However, due to the quite small distance between the slices taken in the range of $0 \mu \mathrm{m}-85 \mu \mathrm{m}$ [27], it is unlikely that the filament paths are deviating to such a large degree locally. Therefore this local deformation is more likely to be an artefact caused by dissimilar magnification during the experiments or a result of the poor input data. As it is not possible to unambiguously determine the source of this deviation, no attempts are made to correct for this local behaviour in this work. The determined properties of the second slice are presented; however, they are not further discussed here.

\subsection{Stiffness}

Treating each filament as individual orthotropic material, the angles of misalignment with the nominal model axis can be used to estimate the resulting mechanical properties 
of each filament in the loading direction [44]. The stiffness of each filament in the nominal longitudinal direction, $E_{x}$, can then, for example, be determined analytically by:

$$
\frac{1}{E_{x}}=\frac{1}{E_{1}} \cos ^{4} \theta+\left(\frac{1}{G_{12}}-\frac{2 v_{12}}{E_{1}}\right) \sin ^{2} \theta \cos ^{2} \theta+\frac{1}{E_{2}} \sin ^{4} \theta
$$

This equation determines the reduction of the nominal longitudinal stiffness, $E_{l}$, by the misalignment and the resulting increasing influence of the transverse stiffness $E_{2}$, shear modulus, $G_{12}$, and the Poison ratio $v_{12}$ on the performance of a filament in loading direction. The carbon fibre properties used in this work are summarised in Table 1 and are taken from the literature [45]. The influence of the matrix material on the tensile properties is small and therefore ignored here. Using equation (1) to predict the individual stiffness of each filament in the nominal direction of the filaments (Figure 8), it is visible that the majority of filament stiffness values are close to the nominal value (Figure 10). It should be noted, that filament cross-sectional shapes and probable varying properties of the filaments are ignored in this graphical representation. The mean tensile stiffness of the system due to filament misalignment is only slightly lower than the nominal stiffness of the filaments, $231 \mathrm{GPa}$ compared to $233 \mathrm{GPa}$. However, as the misalignment is not uniform, areas of lower a higher stiffness form locally (Figure 10). This can lead to different deformation of the material during loading and result in local stress concentrations which may initiate failure. The misalignment in the analysed sample (Figure 8) is rather small. Only one filament deviates more significantly from the nominal direction resulting in a local mismatch in stiffness of about $4.3 \%$ (see minimum value at $-185 \mu \mathrm{m}$ ). It is possible, however, that the misalignments are more significant in a fibre bundle (Figure 1A). This will lead to larger differences in stiffness values and hence, will result in local stress concentrations 
in the material.

\subsection{Strength}

The length of the analysed volume in Mansfield's work [27] is very small ( $\sim 0.5 \mathrm{~mm})$. Stiffness deviations in a larger sample are expected to be more significant. Strength values, especially in compression, will be affected more significantly by filament misalignments. The filament misalignment in respect to the nominal direction reduces the strength of the material. Differences in orientation of neighbouring filaments within a fibre bundle can lead to local stress-concentrations. To estimate the strength, $\sigma_{x}$, as a function of filament misalignment, the Tsai-Hill criterion [44] can be used:

$$
\frac{1}{\sigma_{x}^{2}}=\frac{1}{X^{2}} \cos ^{4} \theta+\left(\frac{1}{S^{2}}-\frac{1}{X^{2}}\right) \sin ^{2} \theta \cos ^{2} \theta+\frac{1}{Y^{2}} \sin ^{4} \theta
$$

This equation relates the strength in the fibre direction, $X$, as a function of the fibre alignment to the transverse, $Y$, and shear strength, $S$. The Tsai-Hill equations were derived to predict strength of entire unidirectional composites. In this work, this equation is used for individual filaments. To incorporate the influence of the matrix material, global composite properties are used in equation (2) for individual filaments (Table 2). These values are taken from the literature for a laminate made from different numbers of pre-impregnated tapes containing $0^{\circ}$ plies only [46].

Using equation (2) and the values in Table 2, the strength can be predicted for the filaments in the individual micrographs (Figure 11); again ignoring possible differences in cross-sectional shapes and properties. Even though the individual stiffness values of the filaments as a function of the misalignment do not deviate significantly (Figure 10), the local differences in strength deviate up to $15 \%$ (Figure 11 at $-515 \mu \mathrm{m}$ ). These local differences in strength may affect the fatigue life of a part in service. A filament 
exhibiting low strength locally, may fracture or initiate a crack. This can then affect the surrounding area and cause crack growth. The analysed area here is very small and the effect of these local misalignments on the entire component cannot be estimated clearly. However, these data can give an idea of local variations of mechanical properties in a fibre bundle. Analysis of larger areas will allow a better understanding of the strength distributions in the future.

\section{Concluding remarks}

A methodology based on the Hungarian algorithm was used to reconstruct filament paths in fibre bundles from two-dimensional micrographs taken at different positions along the bundle path. This technique allows the use of conventional optical imaging techniques, such as microscopy, to analyse the three-dimensional filament arrangement in fibre bundles at higher resolutions compared to micro computed tomography. The sample preparation process of repeatedly removing a small amount of material at the sample surface is destructive; however, it is possible to examine larger areas in an inexpensive way. The amount of manual intervention during the experiments is more demanding compared to micro-CT and the computational time increases with increasing sample size. This technique was demonstrated on a small example taken from the literature for which the point matching and geometrical reconstruction took seconds to complete. This shows that it is possible to estimate changes in mechanical properties easily which can be used as input for more sophisticated numerical models. Future work will aim at demonstrating the applicability of the method for larger samples addressing the assumption in Section 3 that filaments are 'well-behaved', this being less likely to be the case as the sample size increases. It is anticipated that new, better quality images allowing the extraction of filament outlines will provide additional 
information which can be used to build on the methods described here and address the issues arising from increased sample sizes.

\section{Acknowledgements}

This work was supported by the Engineering and Physical Sciences Research Council [grant number: EP/IO33513/1], through the EPSRC Centre for Innovative Manufacturing in Composites (CIMComp) and K.Wedgwood and F.Gommer were supported by separate EPSRC doctoral prize awards hosted by the University of Nottingham. The authors like to thank Dr Martin Corfield from the Power Electronics, Machines and Control Group at the University of Nottingham for the use of the XRADIA Versa XRM-500 system.

\section{References}

[1] Gommer F, Brown LP, Brooks R. Quantification of meso-scale variability and geometrical reconstruction of a textile. Composite Structures. 2015;submitted.

[2] Vanaerschot A, Cox BN, Lomov SV, Vandepitte D. Stochastic framework for quantifying the geometrical variability of laminated textile composites using microcomputed tomography. Compos Part A. 2013;44:122-31.

[3] Endruweit A, Long AC, Robitaille F, Rudd CD. Influence of stochastic fibre angle variations on the permeability of bi-directional textile fabrics. Compos Part A. 2006;37(1):122-32.

[4] Wisnom MR, Atkinson JW. Fibre waviness generation and measurement and its effect on compressive strength. J Reinf Plast Comp. 2000;19(2):96-110.

[5] Requena G, Fiedler G, Seiser B, Degischer P, Di Michiel M, Buslaps T. 3DQuantification of the distribution of continuous fibres in unidirectionally reinforced composites. Compos Part A. 2009;40(2):152-63. 
[6] Rohatgi V, Patel N, Lee LJ. Experimental investigation of flow-induced microvoids during impregnation of unidirectional stitched fiberglass mat. Polym Compos. 1996;17(2):161-70.

[7] Gehrig F, Mannov E, Schulte K. Degradation of NCF-Epoxy Composites containing Voids. 17th International Conference on Composite Materials (ICCM17). Edinburgh, UK, 2009.

[8] Wright P, Fu X, Sinclair I, Spearing SM. Ultra High Resolution Computed Tomography of Damage in Notched Carbon Fiber-Epoxy Composites. J Compos Mater. 2008;42(19):1993-2002.

[9] Gebart BR. Permeability of unidirectional reinforcements for RTM. J Compos Mater. 1992;26(8):1100-33.

[10] Wongsto A, Li S. Micromechanical FE analysis of UD fibre-reinforced composites with fibres distributed at random over the transverse cross-section. Compos Part A. 2005;36(9):1246-66.

[11] Yazdchi K, Srivastava S, Luding S. Micro-macro relations for flow through random arrays of cylinders. Compos Part A. 2012;43(11):2007-20.

[12] Trias D, Costa J, Mayugo JA, Hurtado JE. Random models versus periodic models for fibre reinforced composites. Comput Mater Sci. 2006;38(2):316-24.

[13] Scott AE, Mavrogordato M, Wright P, Sinclair I, Spearing SM. In situ fibre fracture measurement in carbon-epoxy laminates using high resolution computed tomography. Compos Sci Technol. 2011;71(12):1471-7.

[14] Pyka G, Kerckhofs G, Schrooten J, Wevers M. The effect of spatial micro-CT image resolution and surface complexity on the morphological 3D analysis of open porous structures. Materials Characterization. 2014;87:104-15. 
[15] De Boever W, Derluyn H, Van Loo D, Van Hoorebeke L, Cnudde V. Data-fusion of high resolution X-ray CT, SEM and EDS for 3D and pseudo-3D chemical and structural characterization of sandstone. Micron. 2015;74:15-21.

[16] Gommer F, Endruweit A, Long AC. Analysis of Filament Arrangements and Generation of Statistically Equivalent Composite Micro-Structures. Compos Sci Technol. 2014;99:45-51.

[17] Spanne P, Raven C, Snigireva I, Snigirev A. In-line holography and phase-contrast microtomography with high energy x-rays. Phys Med Biol. 1999;44(3):741.

[18] Matveev MY, Long AC, Jones IA. Stochastic modelling of the effect of yarn path and layer shift variability on stiffness and strength of textile composites. 16th European Conference on Composite Materials (ECCM16). Seville, Spain, 2014.

[19] Naouar N, Vidal-Sallé E, Schneider J, Maire E, Boisse P. Meso-scale FE analyses of textile composite reinforcement deformation based on X-ray computed tomography. Composite Structures. 2014;116:165-76.

[20] Straumit I, Lomov SV, Wevers M. Quantification of the internal structure and automatic generation of voxel models of textile composites from X-ray computed tomography data. Compos Part A. 2015;69:150-8.

[21] Guild FJ, Summerscales J. Microstructural image analysis applied to fibre composite materials: a review Comp. 1993;24(5):383-93.

[22] Zangenberg J, Larsen JB, Østergaard RC, Brøndsted P. Methodology for characterisation of glass fibre composite architecture. Plast Rubber Compos. 2012;41(45):187-93.

[23] Paciornik S, D'Almeida JRM. Measurement of Void Content and Distribution in Composite Materials through Digital Microscopy. J Compos Mater. 2009;43(2):101-12. 
[24] Kuhn HW. The Hungarian method for the assignment problem. Nav Res Logist Quart. 1955;2:83-97.

[25] Yurgartis SW, Morey K. Measurement of yarn shape and nesting in plain-weave composites. Compos Sci Technol. 1993;46(1):39-50.

[26] Paluch B. Analysis of geometric imperfections affecting the fibers in unidirectional composites. J Compos Mater. 1996;30(4):454-85.

[27] Mansfield EH, Purslow FRS, Purslow D. The influence of fibre waviness on the moduli of unidirectional fibre reinforced composites. RAE Technical Report1974.

[28] Long AC, Brown LP. Modelling the geometry of textile reinforcements for composites: TexGen. In: Boisse P, editor. Composite reinforcements for optimum performance. Cambridge: Woodhead Publishing Ltd.; 2011.

[29] Burkard R, Dell'Amico M, Martello S. Assignment Problems. 2nd ed. Philadelphia, USA: Society for Industrial and Applied Mathematics; 2012.

[30] Konstantinova P, Udvarev A, Semerdjiev T. A study of a target tracking algorithm using global nearest neighbor approach. 4th international conference on Computer systems and technologies: e-Learning (CompSysTech). New York, USA, 2003. [31] Pardalos PM, Pitsoulis LS. Nonlinear Assignment Problems: Algorithms and Applications. 2001 ed. Dordrecht, NL: Kluwer Academic Pubslishers; 2000.

[32] Poore AB, Gadaleta S. Some assignment problems arising from multiple target tracking Math Comput Model. 2006;43(9-10):1074-91.

[33] Nauss RM. Solving the generalized assignment problem: An optimizing and heuristic approach. Informs J Comput. 2003;15(3):249-66

[34] Jonker R, Volgenant AA. A shortest augmenting path algorithm for dense and sparse linear assignment problems. Computing. 1987;38(4):325-40. 
[35] Salman A, Ahmad I, Al-Madani S. Particle swarm optimization for task assignment problem Microprocess Microsy. 2002;26(8):363-71.

[36] Kuhn HW. Variants of the Hungarian method for assignment problems. Nav Res Logist Quart. 1956;3:253-8.

[37] Munkres J. Algorithms for the assignment and transportation problems. J Soc Ind Appl Math. 1957;5(1):32-8.

[38] Weng S-K, Kuo C-M, Tu S-K. Video object tracking using adaptive Kalman filter. J Vis Commun Image R. 2006;17(6):1190-208.

[39] Kalman RE. A new approach to linear filtering and prediction problems. ASME Journal of Basic Engineering. 1960;82(Series D):35-45.

[40] Kalman RE, Bucy RS. New results in linear filtering and prediction theory. ASME - Journal of Basic Engineering. 1961:95-108.

[41] Cao Y. Hungarian algorithm for linear assignment problems (V2.3). http://uk.mathworks.com/matlabcentral/fileexchange/20652-hungarian-algorithm-forlinear-assignment-problems--v2-3- 04. Last accessed Mar. 2015. [42] Drummond OE, Castañon DA, Bellovin MS. Comparison of 2-D Assignment Algorithms for Sparse, Rectangular, Floating Point, Cost Matrices. Journal of the SDI Panels on Tracking, Institute for Defense Analyses. 1990;4:4-81 to 4-97.

[43] The University of Nottingham. TexGen. http://texgen.sourceforge.net/. Last accessed May 2015.

[44] Jones RM. Mechanics of composite materials: Hemisphere Publishing Corporation; 1975.

[45] Dean GD, Turner P. The elastic properties of carbon fibres and their composites. Comp. 1973;4(4):174-80. 
[46] Lee J, Soutis C. A study on the compressive strength of thick carbon fibre-epoxy laminates. Compos Sci Technol. 2007;67(10):2015-26. 


\section{Figures}

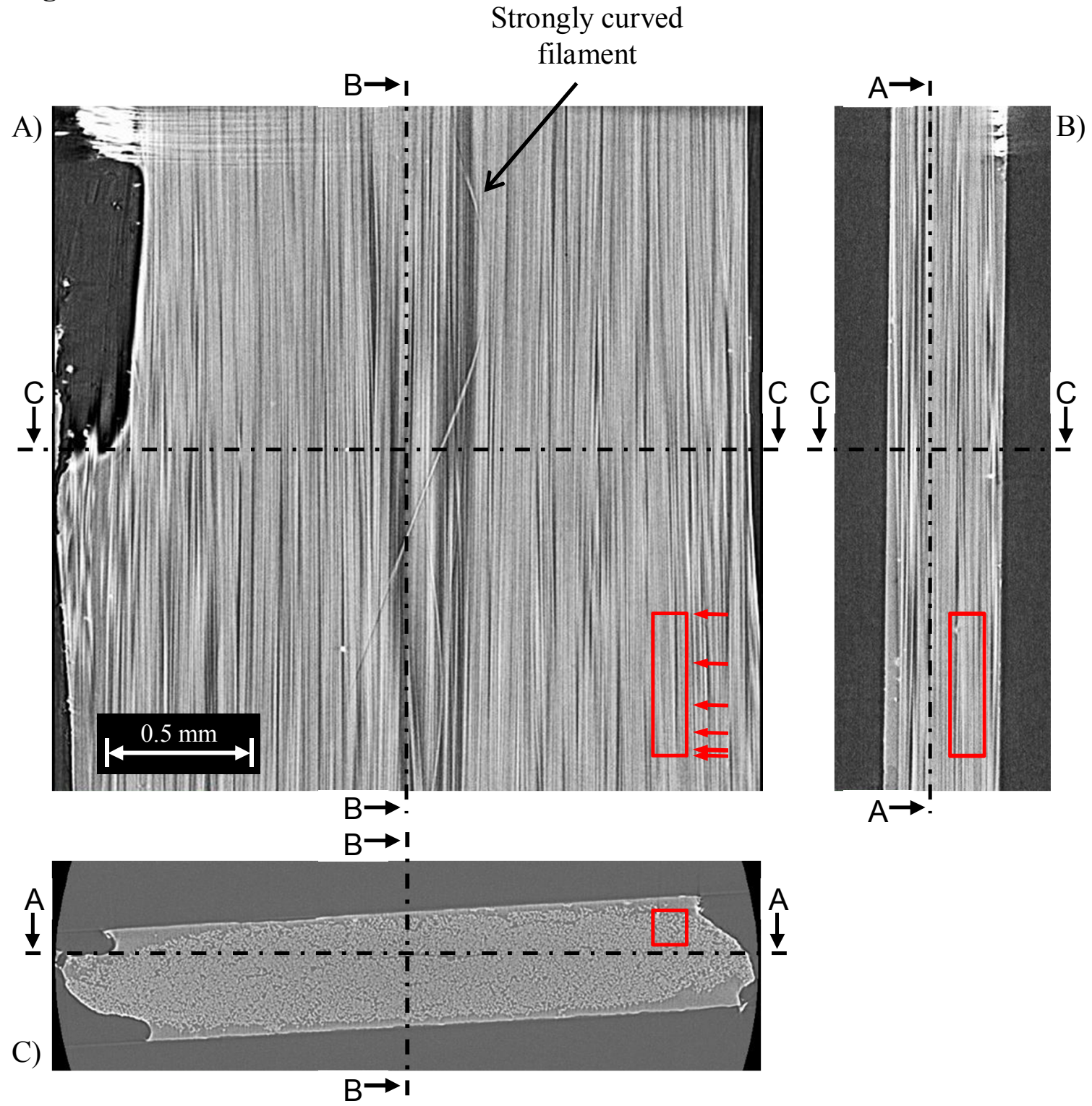

Figure 1: Three sections of a micro-CT image of a $12 \mathrm{~K}$ carbon fibre bundle imaged at a $2.5 \mu \mathrm{m}$ voxel resolution. A schematic of the area analysed by Mansfield [25] is indicated with red rectangles, and slice positions with red arrows in the image. (For interpretation of the references to colour in this figure legend, the reader is referred to the web version of this article.) 


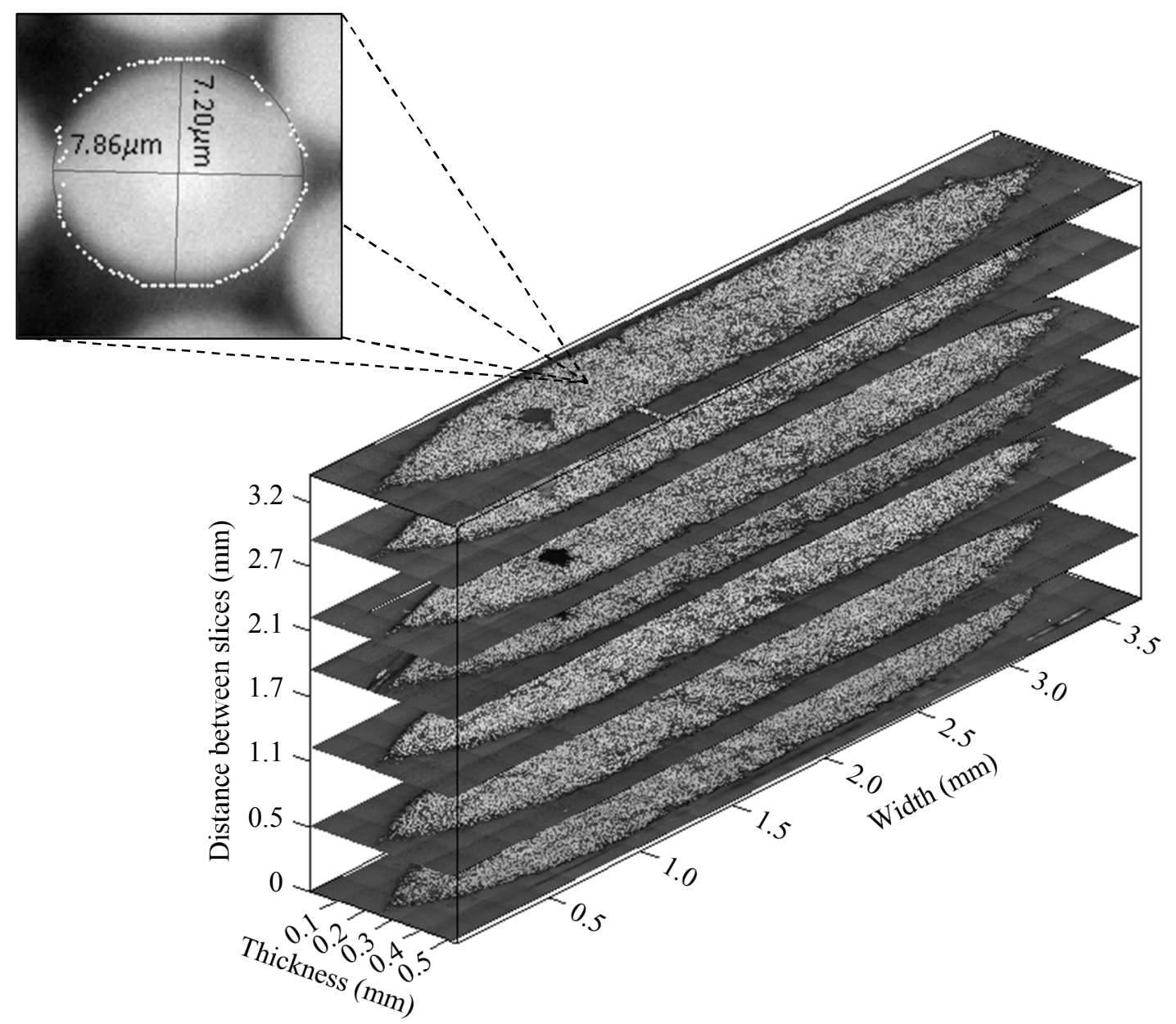

Figure 2: Carbon fibre bundle cross-sections tiled from a number of individual optical micrographs taken along the path of a $12 \mathrm{~K}$ bundle with a resolution of $0.09 \mu \mathrm{m} /$ pixel. The inset shows an individual filament cross-section to demonstrate details visible in each bundle cross-section.

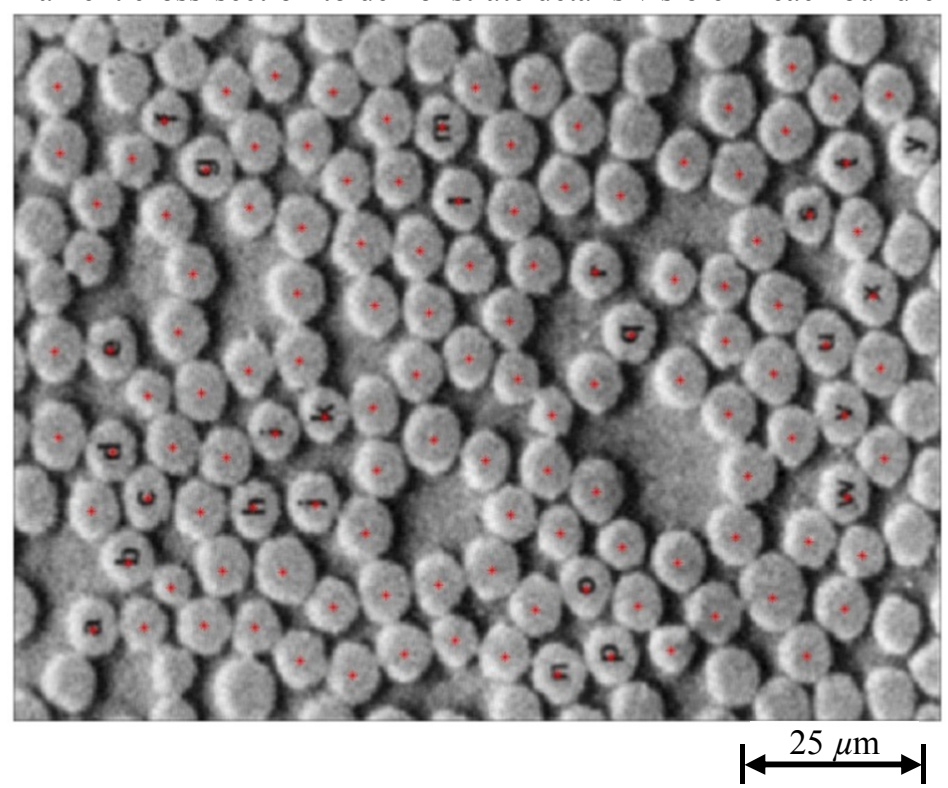

Figure 3: Section of a fibre bundle with marked centre points of the filament cross-sections $(\sim 0.015$ $\mathrm{mm}^{2}$ ). Figure reproduced from Mansfield [25]. (For interpretation of the references to colour in this figure legend, the reader is referred to the web version of this article.) 


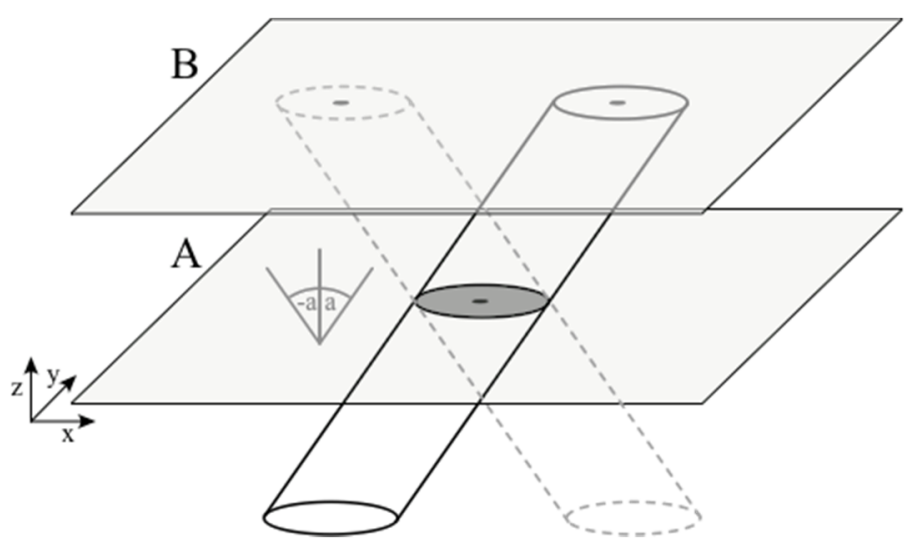

Figure 4: From the eccentricity of the elliptical cross-section when observed through plane $A$, the angle, $a$, which a cylindrical filament makes with the vertical can be obtained. From here, the intersection point of the filament through cross section $B$ can be estimated (thick black fibre); however, this is not unique, as an angle of $-a$ with the vertical is also a possible orientation (grey dashed line).

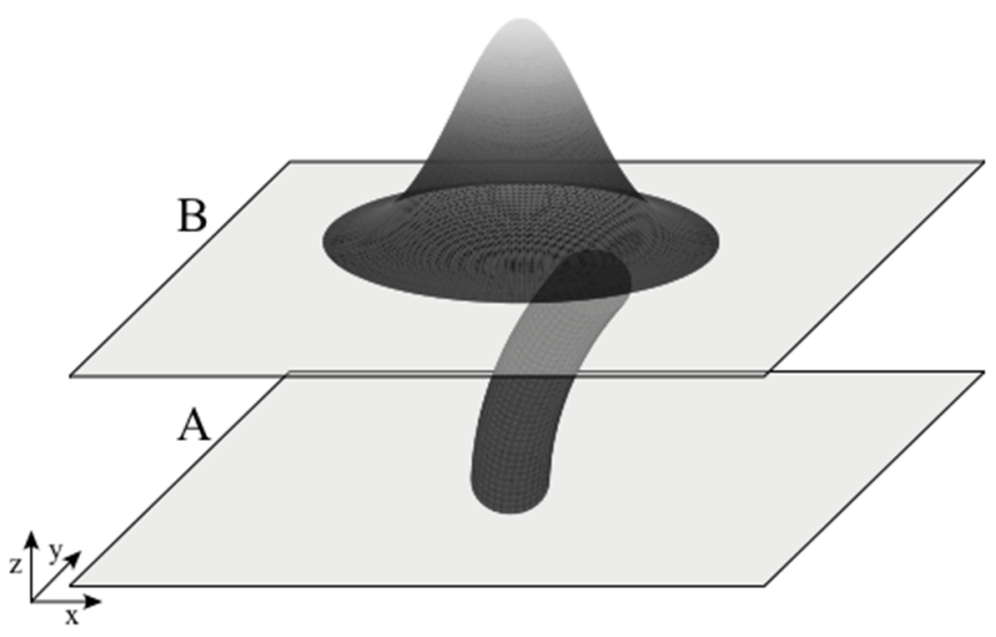

Figure 5: As filaments are not necessarily orientated in straight lines but can bend, the location of their intersection with $B$ is not at the same point as $A$. The bivariate Gaussian distribution superimposed on cross section $B$ shows how the variation from the idealised rod-like orientation is captured in the cost function. 


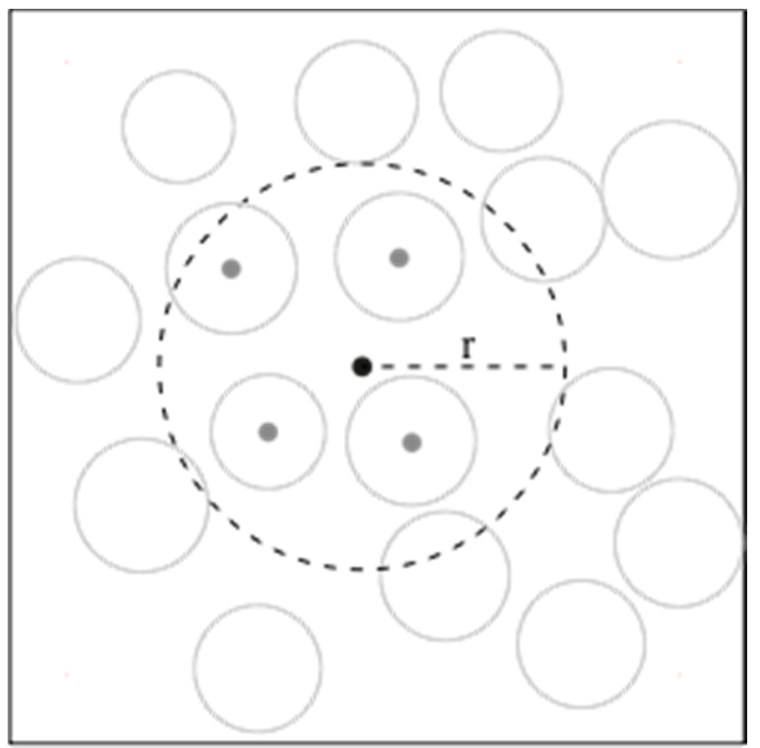

Figure 6: A search radius, $r$, centred at the location of the intersection of a given filament at the previous cross section (black dot), is defined such that the filament can only pass through points inside this circle (grey dots).
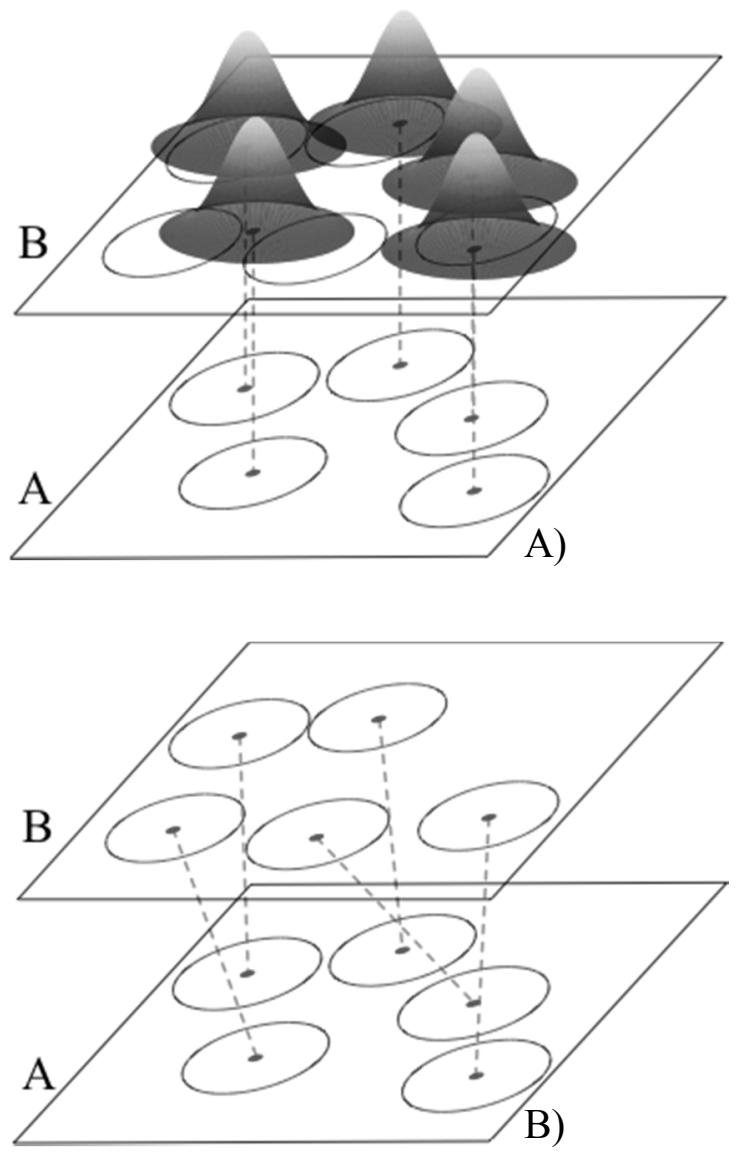

Figure 7: The setup for the example in the text. A) Points in plane $A$ are to be matched with points in $B$ based on the filament centres. Gaussian distributions with a scaled identity covariance matrix, superimposed on cross section $B$ for reference, are used in the construction of the cost matrix. B) Using the Hungarian algorithm, as done in the text, an optimal assignment is guaranteed. 

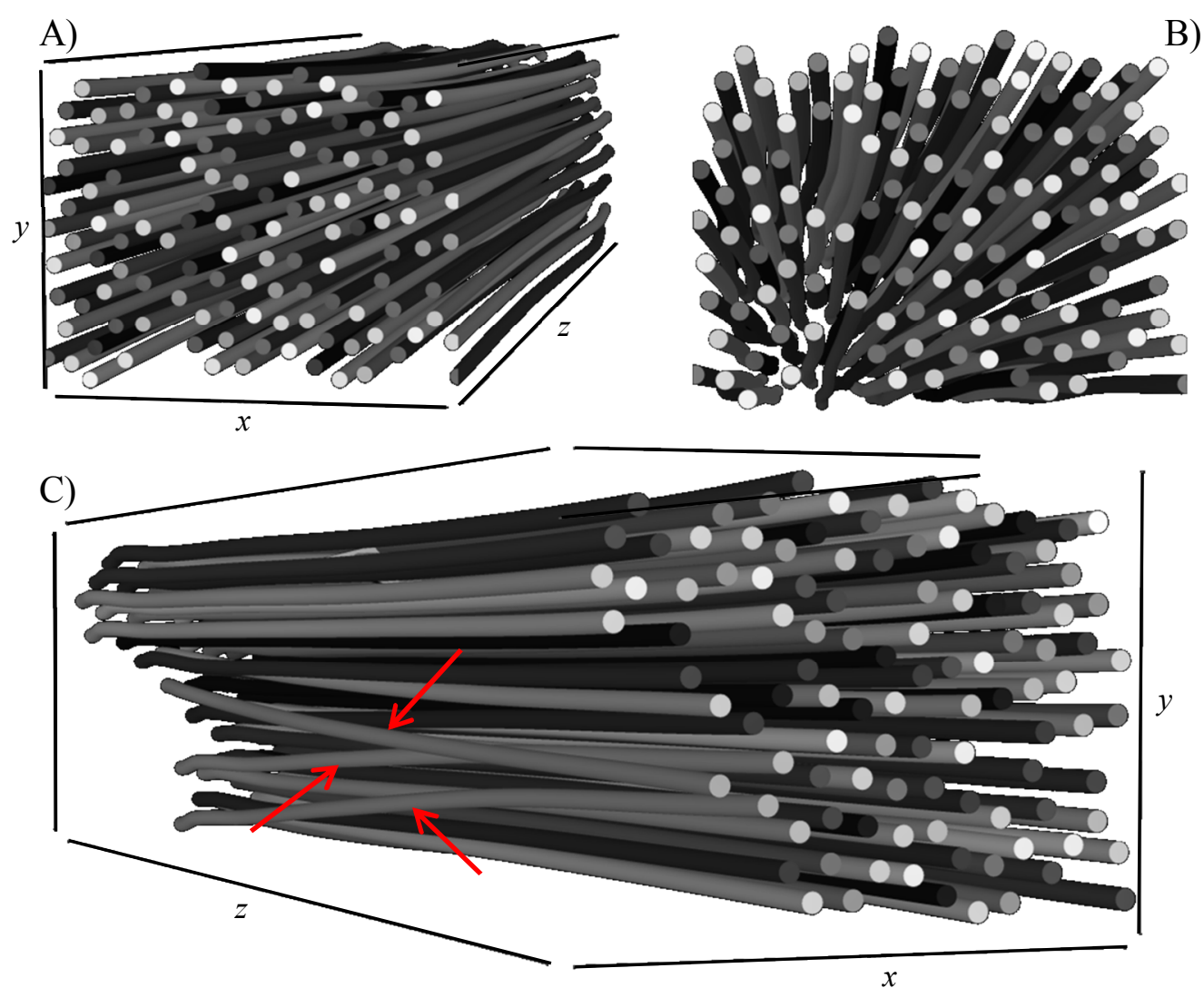

Figure 8: Geometrical model of reconstructed filament created in TexGen [41]. A) Side view, B) front view and $\mathrm{C}$ ) a side view with some filaments removed to highlight internal misalignment. (For interpretation of the references to colour in this figure legend, the reader is referred to the web version of this article.)

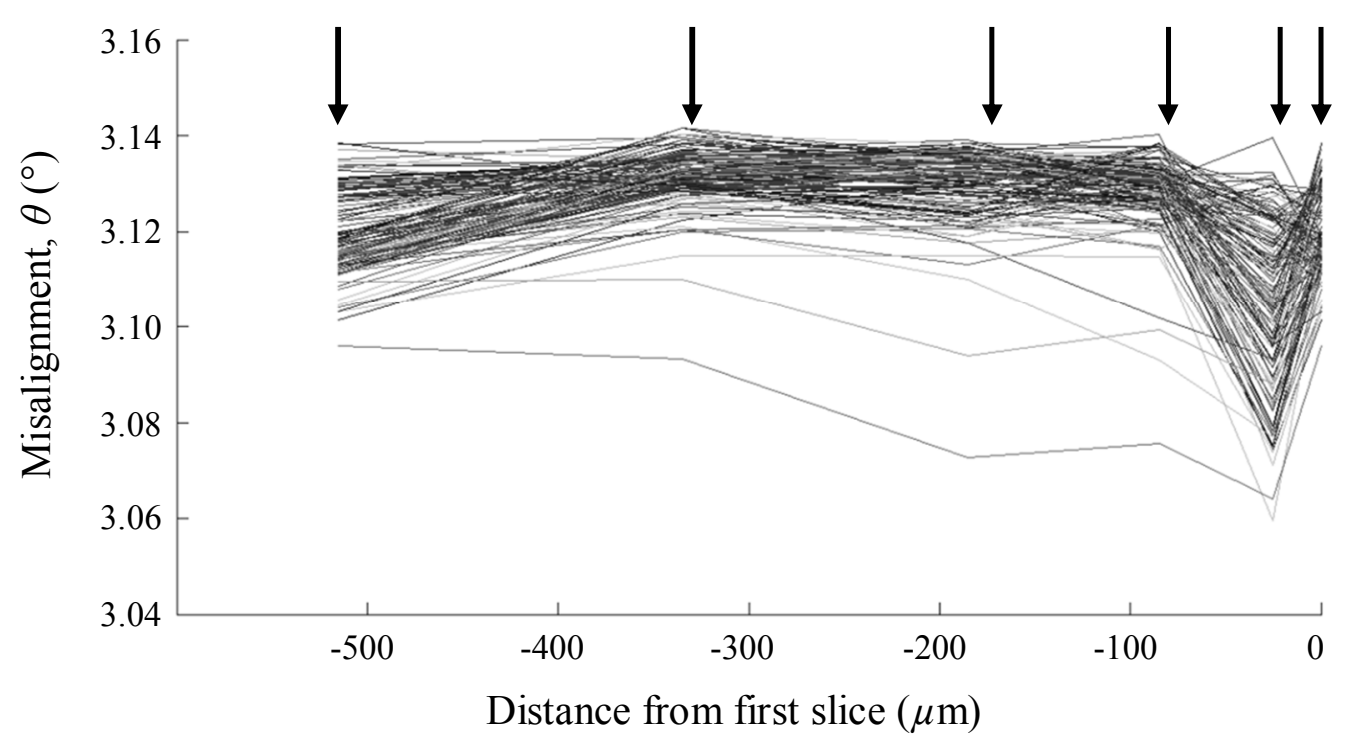

Figure 9: Determined angle, $\theta$, of the filament paths in respect to the nominal model orientations. The relative positions of the micrographs in the sample are indicated by arrows. 

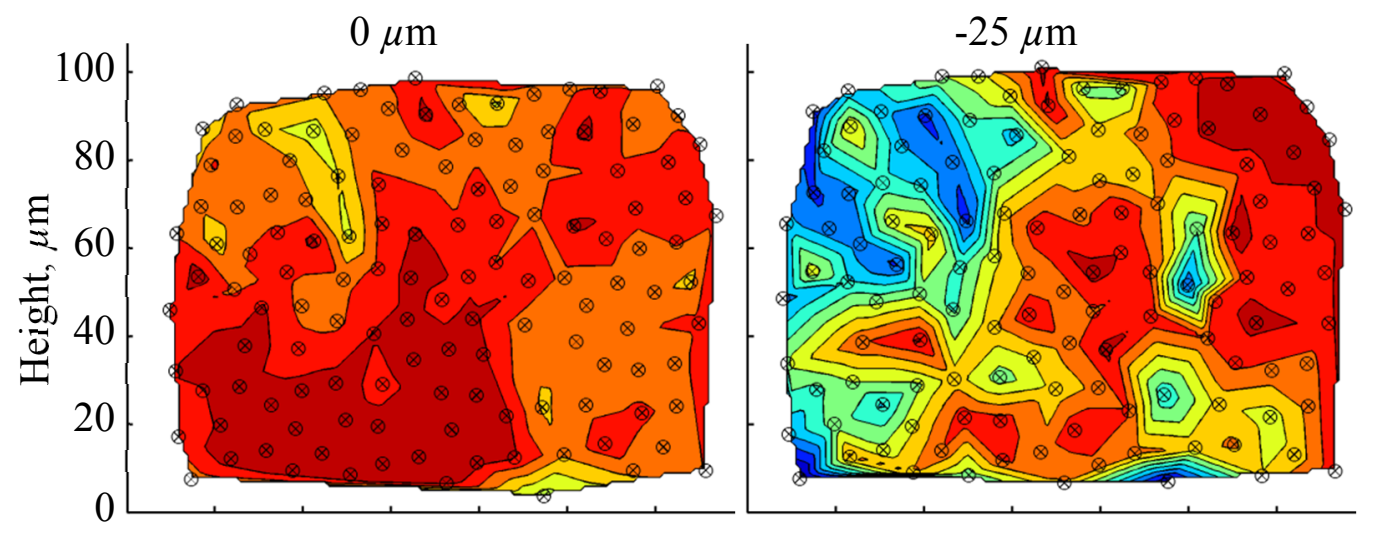

GPa
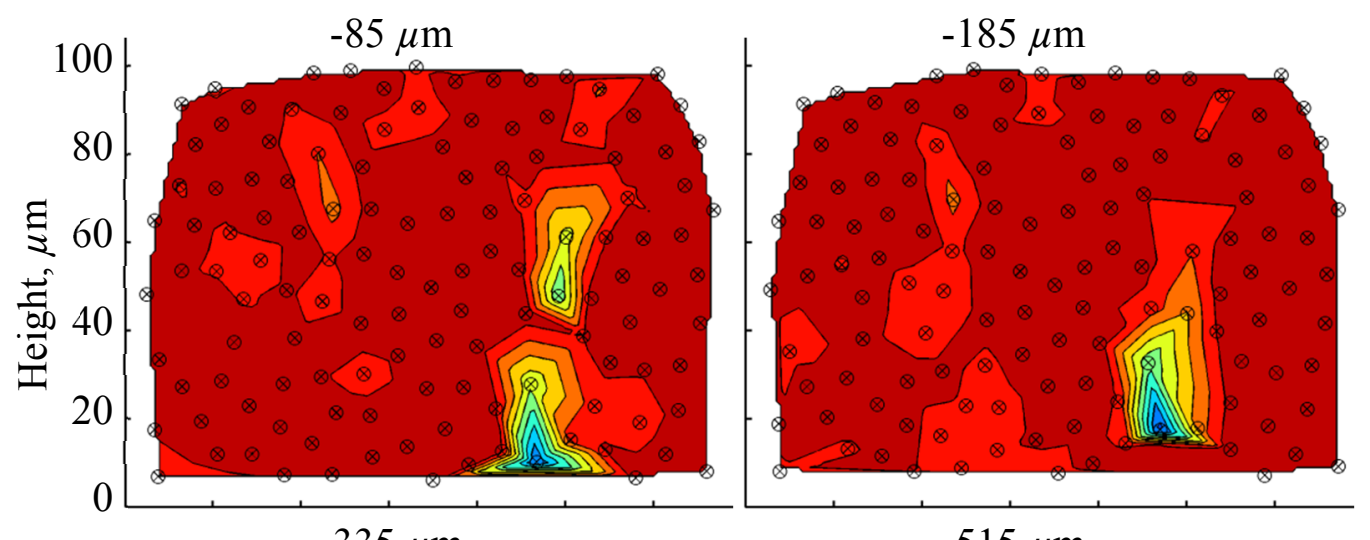

232

230

228

226

224
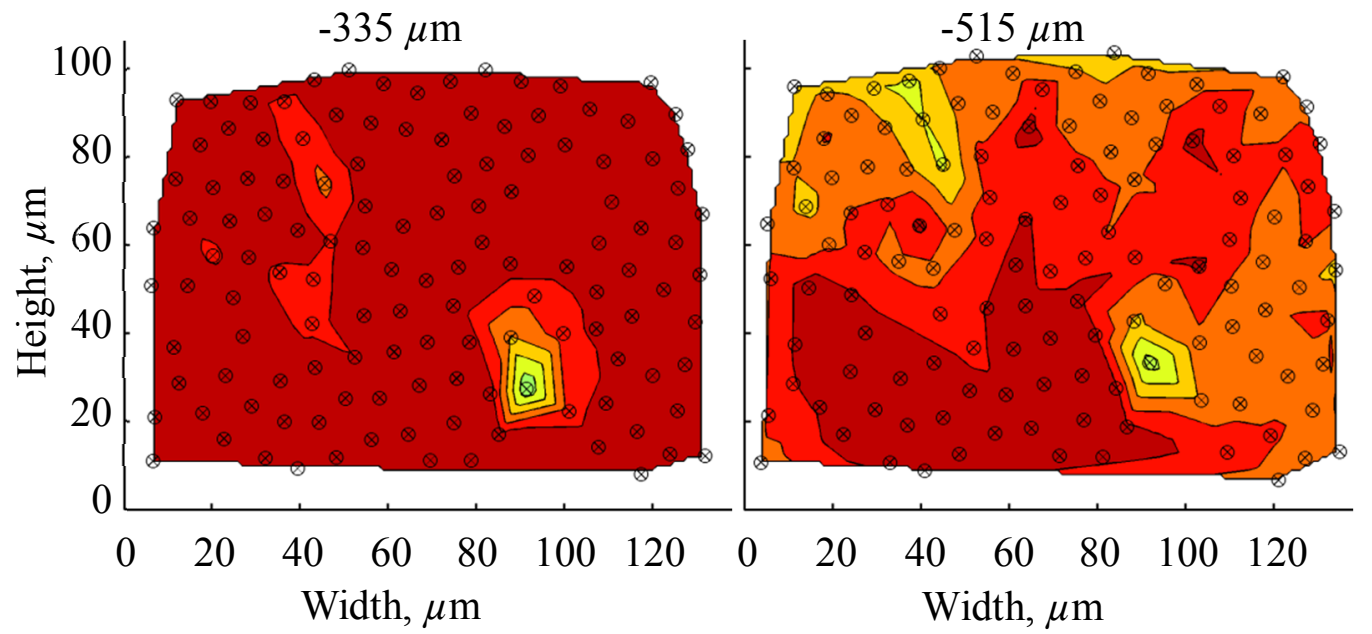

Figure 10: Analytically determined stiffness distributions of the filament arrangement reported in Mansfield [25] based on the filament orientations of the TexGen model in Figure 8. The dots represent filament positions; cross-sectional shapes and matrix material are ignored. (For interpretation of the references to colour in this figure legend, the reader is referred to the web version of this article.) 

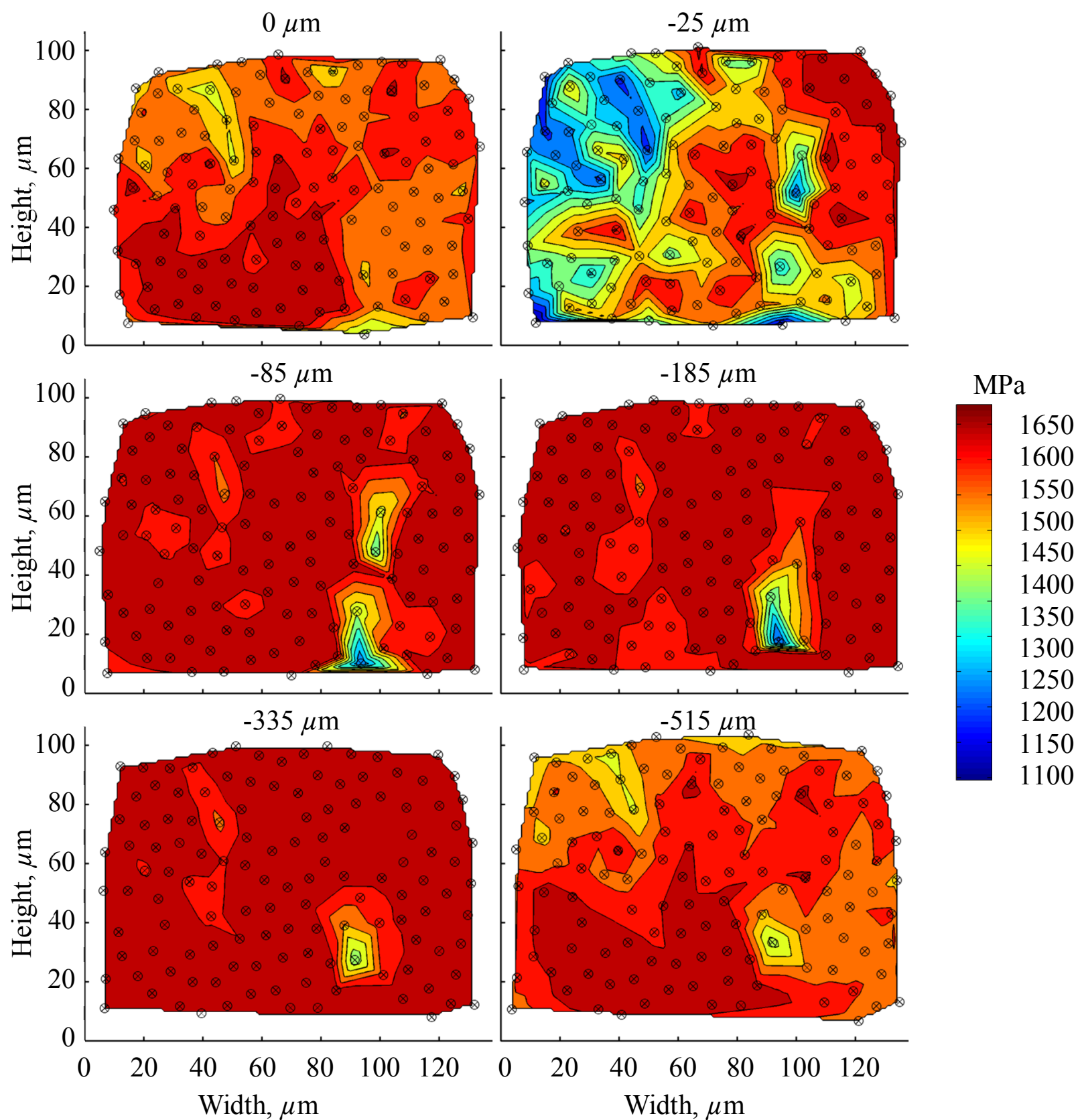

Figure 11: Maps of filament strength distributions in the different slices determined by Tsai-Hill's failure criterion. The dots represent filament positions; cross-sectional shapes and matrix material are ignored. (For interpretation of the references to colour in this figure legend, the reader is referred to the web version of this article.) 


\section{Tables}

Table 1: Nominal stiffness, $E$, shear, $G$, and Poisson ratio, $v$, of single carbon filaments [43]. The subscripts 1 and 2 denote longitudinal and transverse filament directions, respectively.

\begin{tabular}{cc} 
Property & Value \\
\hline$E_{1}$ & $233 \mathrm{GPa}$ \\
$E_{2}$ & $16 \mathrm{GPa}$ \\
$G_{12}$ & $24 \mathrm{GPa}$ \\
$v_{12}$ & 0.35 \\
\hline
\end{tabular}

Table 2: Compressive strength properties in longitudinal, $X$, and transverse, $Y$, direction to the filament paths and the shear strength, $S$, of a unidirectional carbon fibre epoxy composite [44].

\begin{tabular}{cc} 
Property & Value \\
\hline$X$ & $1690 \mathrm{MPa}$ \\
$Y$ & $250 \mathrm{MPa}$ \\
$S$ & $120 \mathrm{MPa}$ \\
\hline
\end{tabular}

